\title{
APPROXIMATION OF RUIN PROBABILITY AND RUIN TIME IN DISCRETE BROWNIAN RISK MODELS
}

\author{
GRIGORI JASNOVIDOV
}

\begin{abstract}
We analyze the classical Brownian risk models discussing the approximation of ruin probabilities (classical, $\gamma$-reflected, Parisian and cumulative Parisian) for the case that ruin can occur only on specific discrete grids. A practical and natural grid of points is for instance $G(1)=\{0,1,2, \ldots\}$, which allows us to study the probability of the ruin on the first day, second day, and so one. For such a discrete setting, there are no explicit formulas for the ruin probabilities mentioned above. In this contribution we derive accurate approximations of ruin probabilities for uniform grids by letting the initial capital to grow to infinity.
\end{abstract}

Key Words: Brownian motion; $\gamma$-reflected risk model; Parisian ruin probability; cumulative Parisian ruin; ruin time approximation

AMS Classification: Primary 60G15; secondary 60G70

\section{INTRODUCTION}

The classical Brownian risk model of an insurance portfolio

$$
R_{u}(t)=u+c t-B(t), \quad t \geq 0,
$$

with $B$ a standard Brownian motion, the initial capital $u>0$ and the premium rate $c>0$, is a key benchmark model in risk theory; see e.g., [1]. For any $u>0$ define the ruin time

$$
\tau(u)=\inf \{t \geq 0: B(t)-c t>u\}
$$

and thus the corresponding ruin probability is given by the well-known formula (see e.g., [2])

$$
\psi_{\infty}(u):=\mathbb{P}\{\tau(u)<\infty\}=\mathbb{P}\left\{\inf _{t \geq 0} R_{u}(t)<0\right\}=e^{-2 c u}
$$

In insurance practice however the ruin probability is relevant not on a continuous time scale, but on a discrete one, due to the operational time (which is discrete). For a given discrete uniform grid $G(\delta)=$ $\{0, \delta, 2 \delta, \ldots\}$ of step $\delta>0$ we define the corresponding ruin probability by

$$
\psi_{\delta, \infty}(u):=\mathbb{P}\left\{\inf _{t \in G(\delta)} R_{u}(t)<0\right\}=\mathbb{P}\left\{\sup _{t \in G(\delta)}(B(t)-c t)>u\right\} .
$$

For any $u>0$ it is not possible to calculate $\psi_{\delta, \infty}(u)$ explicitly and no formulas are available for the distributional characteristics of the corresponding ruin time which we shall denote by $\tau_{\delta}(u)$.

A natural question when explicit formulas are lacking is how can we approximate $\psi_{\delta, \infty}(u)$ and $\tau_{\delta}(u)$ for large $u$ ? Also of interest is to know what is the role of $\delta$ : does it influence the ruin probability in this classical risk model? The first question has been considered recently in [3] for fractional Brownian motion risk process.

Date: January 29, 2020. 
When dealing with the Brownian risk model, both the independence of increments and the self-similarity property are crucial. In particular, those properties are the key to a rigorous and (relatively) simple proof.

Our first result presented next shows that the grid plays a role only with respect to the pre-factor specified by some constant. Specifically, that constant is well-known in the extremes of Gaussian processes being the Pickands constant $\mathcal{H}_{2 c^{2} \delta}$, where

$$
\mathcal{H}_{\eta}=\frac{1}{\eta} \mathbb{E}\left\{\frac{\sup _{t \in \eta \mathbb{Z}} e^{W(t)}}{\sum_{t \in \eta \mathbb{Z}} e^{W(t)}}\right\}=\frac{1}{\eta} \mathbb{E}\left\{\max _{t \geq 0, t \in \eta \mathbb{Z}} e^{W(t)}-\max _{t \geq \eta, t \in \eta \mathbb{Z}} e^{W(t)}\right\} \in(0, \infty)
$$

for any $\eta>0$, with $W(t)=\sqrt{2} B(t)-|t|$. Note that $\mathcal{H}_{0}=1$; the first formula in (3) is derived in [4], whereas the second in [5]. In the following $\sim$ stands for asymptotic equivalence as $u \rightarrow \infty$.

Theorem 1.1. For any $\delta>0$ we have

$$
\psi_{\delta, \infty}(u) \sim \mathcal{H}_{2 c^{2} \delta} \psi_{\infty}(u), \quad u \rightarrow \infty
$$

and further for any $s \in \mathbb{R}$ with $\Phi$ being the distribution function of a standard Gaussian random variable

$$
\lim _{u \rightarrow \infty} \mathbb{P}\left\{c^{3 / 2}\left(\tau_{\delta}(u)-u / c\right) / \sqrt{u} \leq s \mid \tau_{\delta}(u)<\infty\right\}=\Phi(s) .
$$

We note that the above results hold for the continuous case too, where the grid $G(\delta)$ is substituted by $[0, \infty)$ i.e., $\delta=0$. For that case (5) follows from [6]. The approximation in (5) shows that the ruin time is not affected by the density of the grid (i.e., it is independent of $\delta$ ) and thus we conclude that the grid influences only the ruin probability. This is not the case for the ruin probability approximated in (4). For the Pickands constants we have, see e.g., [4, 7, 8]

$$
\mathcal{H}_{2 c^{2} \delta} \leq \mathcal{H}_{0}=1=\lim _{\delta \downarrow 0} \mathcal{H}_{2 c^{2} \delta}=\lim _{c \downarrow 0} \mathcal{H}_{2 c^{2} \delta} .
$$

In particular we see that via self-similarity in the Brownian risk model the role of the grid is coupled with the premium rate $c>0$.

The objective of Section 2 is to explain in detail the main ideas and techniques adequate for the classical Brownian risk model. Section 3 discusses the ruin probability for the $\gamma$-reflected Brownian risk model, see also [9-12]. The approximation of Parisian ruin (see [13-15]) and cumulative Parisian ruin (see [16-18]) is the topic of Section 4. Our findings show that also for those ruin probabilities, the influence of the grid, i.e., the choice of $\delta$ concerns only the leading constant in the asymptotic expansion being further coupled with the premium rate. Given the technical nature of several proofs, we shall relegate them to Section 5, which is followed by an Appendix containing auxiliary calculations.

\section{Approximation techniques for Brownian Risk Model}

Both the independence of increments and the self-similarity property of the Brownian motion render the Brownian risk model very tractable. In order to approximate $\psi_{\delta, \infty}(u)$ for given $\delta>0$ we start with the following lower bound

$$
\psi_{\delta, \infty}(u)=\mathbb{P}\{\exists t \in G(\delta): B(t)>u+c t\} \geq \mathbb{P}\left\{B\left(u t_{u}\right)>u\left(1+c t_{u}\right)\right\}
$$

valid for $t_{u}$ such that $u t_{u} \in G(\delta)$ for all $u$ large. It is clear that such $t_{u}$ exists and moreover

$$
t_{u}=\frac{1}{c}+\frac{\theta_{u}}{u} \in G(\delta)
$$


holds for some $\theta_{u} \in[0, \delta)$ and all large $u$. Consequently, by the well-known Mill's ratio asymptotics $\bar{\Phi}(u) \sim \varphi(u) / u$ as $u \rightarrow \infty$ we obtain for all large $u$ and some positive constant $C$

$$
\psi_{\delta, \infty}(u) \geq \bar{\Phi}\left(\sqrt{u / t_{u}}\left(1+c t_{u}\right)\right) \geq \frac{C}{\sqrt{u}} e^{-2 c u},
$$

where $\bar{\Phi}=1-\Phi$ and $\varphi=\Phi^{\prime}$. Although the lower bound above is not precise enough, it is useful to localize a short interval around

$$
t_{0}:=1 / c
$$

that will lead eventually to the exact approximation of the ruin probability. Indeed, we have with

$$
T_{u}^{ \pm}=u\left(t_{0} \pm u^{-1 / 2} \ln u\right), \quad Z(t)=B(t)-c t
$$

for all large $u$ and any $C>0, p<0$ (the proof is given in the Appendix)

$$
\mathbb{P}\left\{\sup _{t \notin\left[T_{u}^{-}, T_{u}^{+}\right]} Z(t)>u\right\} \leq C u^{p} e^{-2 c u} .
$$

Since for any $u>0$

(9) $\mathbb{P}\left\{\sup _{t \in\left[T_{u}^{-}, T_{u}^{+}\right] \cap \delta \mathbb{Z}} Z(t)>u\right\} \leq \psi_{\delta, \infty}(u) \leq \mathbb{P}\left\{\sup _{t \in\left[T_{u}^{-}, T_{u}^{+}\right] \cap \delta \mathbb{Z}} Z(t)>u\right\}+\mathbb{P}\left\{\sup _{t \notin\left[T_{u}^{-}, T_{u}^{+}\right]} Z(t)>u\right\}$

by (7) and (8) we obtain that $\left(\operatorname{set} \Delta_{\delta}(u)=\left[t_{u}-u^{-1 / 2} \ln u, t_{u}+u^{-1 / 2} \ln u\right] \cap \frac{\delta}{u} \mathbb{Z}\right)$

$$
\psi_{\delta, \infty}(u) \sim \mathbb{P}\left\{\sup _{t \in\left[T_{u}^{-}, T_{u}^{+}\right], t \in \delta \mathbb{Z}} Z(t)>u\right\}=\mathbb{P}\left\{\exists t \in \Delta_{\delta}(u): B(t)>\sqrt{u}(1+c t)\right\}=: P_{\delta}(u), \quad u \rightarrow \infty,
$$

where for the last equality we used the self-similarity property of Brownian motion.

In order to approximate $P_{\delta}(u)$ as $u \rightarrow \infty$ a common approach is to partition $\Delta_{\delta}(u)$ in small intervals and use Bonferroni inequality in order to determine the main contribution to the asymptotics. This idea coupled with the continuous mapping theorem is essentially due to Piterbarg, see e.g., [19]. In this paper we use a modified approach in order to tackle some uniformity issues which arise in the approximations. In particular, we do not use continuous mapping theorem but rely instead on the independence of increments and self-similarity property of Brownian motion. We illustrate below briefly our approach.

We choose a partition $\Delta_{j, S, u},-N_{u} \leq j \leq N_{u}$ of $\Delta_{\delta}(u)$ depending on some constant $S>0$ as follows

$$
\Delta_{j, S, u}=\left[t_{u}+j S u^{-1}, t_{u}+(j+1) S u^{-1}\right] \cap \frac{\delta}{u} \mathbb{Z}, \quad N_{u}=\left\lfloor S^{-1} \ln (u) \sqrt{u}\right\rfloor .
$$

Here $\lfloor\cdot\rfloor$ stands for the ceiling function. The Bonferroni inequality yields

$$
p_{1}(S, u) \geq P_{\delta}(u) \geq p_{1}^{\prime}(S, u)-p_{2}(S, u)
$$

where

$$
p_{1}(S, u)=\sum_{j=-N_{u}-1}^{N_{u}} p_{j, S, u}, \quad p_{1}^{\prime}(S, u)=\sum_{j=-N_{u}}^{N_{u}-1} p_{j, S, u}, \quad p_{2}(S, u)=\sum_{-N_{u}-1 \leq j<i \leq N_{u}} p_{i, j ; S, u},
$$

with

$p_{j, S, u}=\mathbb{P}\left\{\exists_{t \in \Delta_{j, S, u}} B(t)>\sqrt{u}(1+c t)\right\} \quad$ and $p_{i, j ; S, u}=\mathbb{P}\left\{\exists_{t \in \Delta_{i, S, u}} B(t)>\sqrt{u}(1+c t), \exists_{t \in \Delta_{j, S, u}} B(t)>\sqrt{u}(1+c t)\right\}$. 
As shown in [20] [Eq. (43)] the term $p_{2}(S, u)$, also referred to as the double-sum term, is negligible compared with $p_{1}^{\prime}(S, u)$ if we let $u \rightarrow \infty$ and then $S \rightarrow \infty$.

Moreover, $p_{1}(S, u)$ and $p_{1}^{\prime}(S, u)$ are asymptotically equivalent with $P_{\delta}(u)$, i.e.,

$$
\lim _{S \rightarrow \infty} \lim _{u \rightarrow \infty} p_{1}(S, u) / p_{1}^{\prime}(S, u)=\lim _{S \rightarrow \infty} \lim _{u \rightarrow \infty} p_{1}(S, u) / P_{\delta}(u)=1 .
$$

The main question is therefore how to approximate $p_{1}(S, u)$ ?

In order to answer the above question we need to approximate each term $p_{j, S, u}$ as $u \rightarrow \infty$. Moreover, such approximation has to be uniform for all $j$ satisfying $-N_{u} \leq j \leq N_{u}$, which is a subtle issue solved in this paper by utilizing the independence of increments of Brownian motion and the self-similarity property; see the proof of Theorem 1.1 in Section 5 and [20] for similar ideas in the continuous time setting.

\section{3. $\gamma$-Reflected Risk Model}

An interesting extension of the classical Brownian risk model is that of $\gamma$-reflected Brownian risk model introduced in [21]. The $\gamma$-reflected fractional Brownian motion risk model and its extensions are discussed in [9-12]. In this section we consider the approximation of the ruin probability over a discrete grid $G(\delta), \delta>0$ for the $\gamma$-reflected Brownian motion model. Specifically, for $\gamma \in(0,1)$ we define the risk model

$$
R_{u}^{\gamma}(t)=u+c t-B(t)+\gamma \inf _{0 \leq s \leq t}(B(s)-c s), \quad t \geq 0
$$

For given $\delta>0$ we are interested in the ruin probability in discrete time, namely

$$
\Upsilon_{\gamma, \delta}(u)=\mathbb{P}\left\{\sup _{t \in[0, \infty) \cap \delta \mathbb{Z}}\left(B(t)-c t-\gamma \inf _{s \in[0, t] \cap \delta \mathbb{Z}}(B(s)-c s)\right)>u\right\},
$$

which cannot be calculated explicitly. The risk process $R_{u}^{\gamma}(t)$ is not Gaussian any more, however using the independence of the increments of Brownian motion and the self-similarity property, for any $u>0$ we have with $t=k-l, s=l$

$$
\begin{aligned}
\Upsilon_{\gamma, \delta}(u) & =\mathbb{P}\{\exists l \leq k \in G(\delta): B(k)-k c-\gamma(B(l)-c l)>u\} \\
& =\mathbb{P}\{\exists l \leq k \in G(\delta):(B(k)-B(l))+(1-\gamma) B(l)-c(k-\gamma l)>u\} \\
& =\mathbb{P}\left\{\exists l \leq k \in G(\delta): B(k-l)+(1-\gamma) B^{*}(l)-c(k-\gamma l)>u\right\} \\
& =\mathbb{P}\left\{\exists t, s \in G(\delta):(B(t)-c t)+(1-\gamma)\left(B^{*}(s)-c s\right)>u\right\} \\
& =\mathbb{P}\left\{\exists t, s \in G(\delta / u): \frac{B(t)+(1-\gamma) B^{*}(s)}{c t+(1-\gamma) c s+1}>\sqrt{u}\right\},
\end{aligned}
$$

where $B^{*}$ is an independent copy of $B$. The above re-formulation shows that the ruin probability concerns the supremum of the random field $Z$ given by

$$
Z(t, s)=\frac{B(t)+(1-\gamma) B^{*}(s)}{c t+(1-\gamma) c s+1}, \quad s, t \geq 0
$$

From [9] it follows, that for any $\eta, a>0$

$$
\mathcal{P}_{\eta}^{a}:=\mathbb{E}\left\{\sup _{t \in[0, \infty) \cap \eta \mathbb{Z}} e^{\sqrt{2} B(t)-t(1+a)}\right\} \in(0, \infty) .
$$

Our next result gives the approximation of the above ruin probability as $u \rightarrow \infty$. 
Theorem 3.1. For any $\delta>0$ and any $\gamma \in(0,1)$

$$
\Upsilon_{\gamma, \delta}(u) \sim \mathcal{P}_{2 c^{2}(1-\gamma)^{2} \delta}^{\frac{\gamma}{1-\gamma}} \mathcal{H}_{2 c^{2} \delta} \psi_{\infty}(u), \quad u \rightarrow \infty
$$

We note that the basic properties of discrete Piterbarg constants are discussed in [8, 22].

\section{Parisian \& Cumulative Parisian Ruin}

4.1. Parisian ruin. In this section we expand our results to the Parisian ruin. For the continuous time [13] gives an exact formula for the Parisian ruin probability. Both finite and infinite Parisian ruin times for continuous setup of the problem are dealt with in $[14,15]$.

Next, for given $\delta, T$ positive (suppose for convenience that $T / \delta \in G(\delta)$ ) define the Parisian ruin for the discrete grid $G(\delta)$ by

$$
\mathcal{P}_{\delta}(u, T)=\mathbb{P}\left\{\inf _{t \in[0, \infty) \cap \delta \mathbb{Z}} \sup _{s \in[t, t+T] \cap \delta \mathbb{Z}} R_{u}(s)<0\right\} .
$$

Our next result shows again that the grid determines the asymptotic approximation via the constant $\mathcal{H}_{\eta, T}$ defined for $\eta, T$ positive by

$$
\mathcal{H}_{\eta, T}=\mathbb{E}\left\{\frac{\sup _{t \in \eta \mathbb{Z}} \inf _{s \in[t, t+T] \cap \eta \mathbb{Z}} e^{\sqrt{2} B(s)-|s|}}{\eta \sum_{t \in \eta \mathbb{Z}} e^{\sqrt{2} B(t)-|t|}}\right\} \in(0, \infty) .
$$

Note that if $T=0$, then $\mathcal{H}_{\eta, 0}$ equals the Pickands constant $\mathcal{H}_{\eta}$ defined in (3). The corresponding constant for the continuous case is introduced in [14].

Theorem 4.1. For any $\delta, T>0$

$$
\mathcal{P}_{\delta}(u, T) \sim \mathcal{H}_{2 c^{2} \delta, 2 c^{2} T} \psi_{\infty}(u), \quad u \rightarrow \infty
$$

We see from the approximation above that the premium rate $c$ influences also the leading constant in the asymptotics.

4.2. Cumulative Parisian ruin. Cumulative Parisian ruin for fractional Brownian motion risk model has been discussed recently in [16]. As therein, adjusted for the discrete setup, we define the cumulative Parisian ruin probability by

$$
\mathcal{C}_{\delta}(u, k)=\mathbb{P}\{\#\{t \in G(\delta): B(t)-c t>u\}>k\},
$$

where $k$ is some non-negative integer and the symbol \# stands for the number of the elements of a given set. Note in passing that $\mathcal{C}_{\delta}(u, 0)=\psi_{\delta, \infty}(u)$. Next, for $\eta>0$ define the constant

$$
\mathcal{B}_{\eta}(k)=\lim _{S \rightarrow \infty} \frac{\mathcal{B}_{\eta}(S, k)}{S}
$$

where for any $S>0$

$$
\mathcal{B}_{\eta}(S, k)=\int_{\mathbb{R}} \mathbb{P}\left\{\eta \sum_{s \in[0, S] \cap \eta \mathbb{Z}} \mathbb{I}(\sqrt{2} B(s)-|s|+z>0)>k\right\} e^{-z} d z,
$$

with $\mathbb{I}(\cdot)$ denoting the indicator function. In view of $[17] \mathcal{B}_{\eta}(k)$ is positive and finite. 
Theorem 4.2. For any non-negative integer $k$ we have as $u \rightarrow \infty$

$$
\mathcal{C}_{\delta}(u, k) \sim \mathcal{B}_{2 c^{2} \delta}(k) \psi_{\infty}(u)
$$

Remark 4.3. i) Defining the ruin times corresponding to Parisian and cumulative Parisian ruin, it follows with similar arguments as in the proof of Theorem 1.1 that those can be approximated in the same way as (5).

ii) If $k=0$, then the claim in (16) reduces to (4).

\section{Proofs}

Proof of Theorem 1.1: As mentioned in Section 2, the negligibility of the double-sum term follows by [20], hence the claim in (4) follows thus by approximating $p_{1}(S, u)$ as $u \rightarrow \infty$. We show first the approximation of $p_{j, S, u}$ as $u \rightarrow \infty$ uniformly for $-N_{u} \leq j \leq N_{u}$. Note that with $u=v^{2}$ and $\mathcal{N}$ being a standard Gaussian random variable we have the distributional representation based on the independence of increments of Brownian motion

$$
B\left(c_{j, S, u}+t / u\right)=\sqrt{c_{j, S, v}} \mathcal{N}+B(t) / v, \quad t \in[0, S], u>0, c_{j, S, v}=t_{u}+j S v^{-2} .
$$

Recall that $t_{u} \in G(\delta)$ is given by $t_{u}=1 / c+\theta_{u} / u$ for some $\theta_{u} \in[0, \delta)$. It turns out that $\theta_{u}$ will not play any role in the final asymptotic approximation. We have thus with $\varphi_{j, v}$ the probability density function of $\sqrt{c_{j, S, v}} \mathcal{N}$

$$
\begin{aligned}
p_{j, S, u} & =\mathbb{P}\left\{\exists_{t \in \Delta_{j, S, u}}:(B(t)-\sqrt{u} c t)>\sqrt{u}\right\} \\
& =\int_{\mathbb{R}} \mathbb{P}\left\{\exists_{t \in[0, S] \cap \delta \mathbb{Z}}:\left(B(t) / v-v c\left(c_{j, S, v}+t / v^{2}\right)>v-x \mid \sqrt{c_{j, S, v}} \mathcal{N}=x\right\} \varphi_{j, v}(x) d x\right. \\
& =\frac{1}{v} \int_{\mathbb{R}} \mathbb{P}\left\{\exists_{t \in[0, S] \cap \delta \mathbb{Z}}:\left(B(t) / v-v c\left(c_{j, S, v}+t / v^{2}\right)>v-(v-x / v)\right\} \varphi_{j, v}(v-x / v) d x\right. \\
& =\frac{1}{v} \int_{\mathbb{R}} \mathbb{P}\left\{\exists_{t \in[0, S] \cap \delta \mathbb{Z}}: Z(t)>x+c c_{j, S, v} v^{2}\right\} \varphi_{j, v}(v-x / v) d x \\
& =\frac{1}{v} \int_{\mathbb{R}} \mathbb{P}\left\{\exists_{t \in[0, S] \cap \delta \mathbb{Z}}: Z(t)>x\right\} \varphi_{j, v}\left(v\left(1+c c_{j, S, v}\right)-x / v\right) d x \\
& =\frac{e^{-v^{2}\left(1+c c_{j, S, v}\right)^{2} /\left(2 c_{j, S, v}\right)}}{v \sqrt{2 \pi c_{j, S, v}}} \int_{\mathbb{R}} w(x) \omega(j, S, x) d x,
\end{aligned}
$$

where $($ recall $Z(t)=B(t)-c t, t \geq 0)$

$$
w(x)=\mathbb{P}\left\{\exists_{t \in[0, S] \cap \delta \mathbb{Z}}: Z(t)>x\right\}, \quad \omega(j, S, x)=e^{x\left(1+c c_{j, S, v}\right) / c_{j, S, v}-x^{2} /\left(2 c_{j, S, v} v^{2}\right)} .
$$

Using Borell-TIS inequality (see e.g., [23]) we have (proof is given in the Appendix)

$$
\int_{\mathbb{R}} w(x) \omega(j, S, x) d x=\int_{-M}^{M} w(x) e^{2 c x} d x+A_{M, v}
$$

where $A_{M, v} \rightarrow 0$ as $u \rightarrow \infty$ and then $M \rightarrow \infty$, uniformly for $-N_{u} \leq j \leq N_{u}$ and $S>0$. By the monotone convergence theorem

$$
\lim _{M \rightarrow \infty} \int_{-M}^{M} w(x) e^{2 c x} d x=\frac{1}{2 c} \mathbb{E}\left\{\sup _{t \in[0, S] \cap \delta \mathbb{Z}} e^{2 c B(t)-2 c^{2} t}\right\}=\frac{1}{2 c} \mathbb{E}\left\{\sup _{t \in[0, S] \cap \delta \mathbb{Z}} e^{\sqrt{2} B\left(2 c^{2} t\right)-2 c^{2} t}\right\}
$$




$$
=\frac{1}{2 c} \mathbb{E}\left\{\sup _{t \in\left[0,2 c^{2} S\right] \cap 2 c^{2} \delta \mathbb{Z}} e^{\sqrt{2} B(t)-t}\right\} .
$$

In a view of the definition of discrete Pickands constants, see e.g., [4, 24]

$$
\lim _{S \rightarrow \infty} \frac{1}{2 c^{2} S} \mathbb{E}\left\{\sup _{t \in\left[0,2 c^{2} S\right] \cap 2 c^{2} \delta \mathbb{Z}} e^{\sqrt{2} B(t)-t}\right\}=\mathcal{H}_{2 c^{2} \delta},
$$

with $\mathcal{H}_{\eta}$ defined in (3). Consequently, the asymptotics of $p_{1}(S, u)$ as $u \rightarrow \infty$ and therefore also (5) follow by calculating the limit as $u \rightarrow \infty, S \rightarrow \infty$ of

$$
K_{v, S}=e^{2 v^{2} c} c S \sum_{j=-N_{u}-1}^{N_{u}} \frac{e^{-v^{2}\left(1+c c_{j, S, v}\right)^{2} /\left(2 c_{j, S, v}\right)}}{v \sqrt{2 \pi c_{j, S, v}}} .
$$

Setting

$$
f(t)=(1+c t)^{2} / 2 t=1 /(2 t)+c+c^{2} t / 2, \quad f^{\prime}(t)=\left(-1 / t^{2}+c^{2}\right) / 2, \quad f^{\prime \prime}(t)=1 / t^{3}
$$

we have that $f^{\prime}\left(t_{0}\right)=0$ implying

$$
f\left(t_{0}+x\right)-f\left(t_{0}\right)=\frac{f^{\prime \prime}\left(t_{0}\right)}{2} x^{2}+O\left(x^{3}\right)
$$

as $x \rightarrow 0$ with $f^{\prime \prime}\left(t_{0}\right)=c^{3}$. Consequently, as $u \rightarrow \infty$

$$
\begin{aligned}
K_{v, S} & \sim \frac{c}{\sqrt{2 \pi t_{0}}} \frac{S}{v} \sum_{j=-N_{u}-1}^{N_{u}} e^{-\left(v^{2} f\left(t_{0}+\left(j S+\theta_{u}\right) / v^{2}\right)-v^{2} f\left(t_{0}\right)\right)} \\
& \sim \frac{c}{\sqrt{2 \pi t_{0}}} \frac{S}{v} \sum_{j=-N_{u}-1}^{N_{u}} e^{-f^{\prime \prime}\left(t_{0}\right)\left(\left(j S+\theta_{u}\right)^{2} / v^{2}\right) / 2} \\
& \sim \frac{c^{3 / 2}}{\sqrt{2 \pi}} \int_{\mathbb{R}} e^{-f^{\prime \prime}\left(t_{0}\right) x^{2} / 2} d x=1,
\end{aligned}
$$

where the last two steps follow with the same arguments as in the proof of (39) in [20]. Finally, we have that as $u \rightarrow \infty$ and then $S \rightarrow \infty$

$$
P_{\delta}(u) \sim K_{v, S} \mathcal{H}_{2 c^{2} \delta} e^{-2 u c} \sim \mathcal{H}_{2 c^{2} \delta} e^{-2 u c} .
$$

We show next (5). For any $u>0, s \in \mathbb{R}$ we have

$$
\mathbb{P}\left\{\tau_{\delta}(u)-u t_{u} \leq s \sqrt{u} \mid \tau_{\delta}(u)<\infty\right\}=\frac{1}{\psi_{\delta, \infty}(u)} \mathbb{P}\left\{\exists_{t \in\left[0, u t_{u}+s \sqrt{u}\right] \cap \delta \mathbb{Z}}: Z(t)>u\right\} .
$$

Considering the approximations of $p_{j, S, u}$ uniformly for all $-N_{u} \leq j \leq N_{u}^{\prime}$ with $N_{u}^{\prime}=\lfloor s \sqrt{u} / S\rfloor$ we obtain as above

$\lim _{u \rightarrow \infty} \frac{1}{\psi_{\delta, \infty}(u)} \mathbb{P}\left\{\exists_{t \in\left[0, u t_{u}+s \sqrt{u}\right] \cap \delta \mathbb{Z}}: Z(t)>u\right\}=\frac{\int_{-\infty}^{s} e^{-f^{\prime \prime}\left(t_{0}\right) x^{2} / 2} d x}{\int_{\mathbb{R}} e^{-f^{\prime \prime}\left(t_{0}\right) x^{2} / 2} d x}=\frac{c^{3 / 2}}{\sqrt{2 \pi}} \int_{-\infty}^{s} e^{-c^{3} x^{2} / 2} d x=\Phi\left(s c^{3 / 2}\right)$.

Hence

$$
\lim _{u \rightarrow \infty} \mathbb{P}\left\{\left(\tau_{\delta}(u)-u t_{u}\right) / \sqrt{u} \leq s \mid \tau_{\delta}(u)<\infty\right\}=\Phi\left(s c^{3 / 2}\right), \quad s \in \mathbb{R} .
$$

Since $\Phi$ is continuous, by Dini's theorem, the above convergence holds also substituting $s$ by $s_{u}$ such that $\lim _{u \rightarrow \infty} s_{u}=s \in \mathbb{R}$. Consequently, since $\theta_{u} \in[0, \delta)$ we have also

$$
\lim _{u \rightarrow \infty} \mathbb{P}\left\{c^{3 / 2}\left(\tau_{\delta}(u)-u t_{0}\right) / \sqrt{u} \leq s \mid \tau_{\delta}(u)<\infty\right\}=\Phi(s), \quad s \in \mathbb{R} .
$$


Proof of Theorem 3.1. Recall that $t_{u}=t_{0}+\theta_{u} / u=1 / c+\theta_{u} / u$ and denote $\beta=1-\gamma$. We analyze the variance function $\sigma_{Z}^{2}$ of the process $Z(t, s)$. For any non-negative $s, t$ we have

$$
\sigma_{Z}^{2}(t, s)=\frac{t+\beta^{2} s}{(c t+\beta c s+1)^{2}}=\frac{t+\beta s}{(c t+\beta c s+1)^{2}}-\frac{\beta(1-\beta) s}{(c t+\beta c s+1)^{2}}=: A(t, s)-A^{*}(t, s) .
$$

Note that $A(t, s)$ depends only on $t+\beta s$ and achieves its global maxima on the line $t+\beta s=t_{0}=1 / c$, while $A^{*}(t, s)$ is negative for all $s>0$ and equals zero for $s=0$. Hence $(t, s)=(1 / c, 0)$ is the unique global maxima of $\sigma_{Z}^{2}(t, s)$ and $\sigma_{Z}^{2}(1 / c, 0)=\frac{1}{4 c}$. We define next

$$
\mathbb{D}_{\delta}(u)=\left\{s, t \in G(\delta / u):(t, s) \in\left(-\frac{\ln u}{\sqrt{u}}+t_{u}, \frac{\ln u}{\sqrt{u}}+t_{u}\right) \times\left(0, \frac{\ln u}{\sqrt{u}}\right)\right\} .
$$

We have (proof see in the Appendix)

$$
\Upsilon_{\gamma, \delta}(u) \sim \mathbb{P}\left\{\exists t, s \in \mathbb{D}_{\delta}(u): Z(t, s)>\sqrt{u}\right\}=: \zeta(u), \quad u \rightarrow \infty .
$$

Let $\Delta_{i, S, u}$ be as in (10) and set

$$
\begin{array}{r}
p(i, j)=\mathbb{P}\left\{\exists t, s \in \Delta_{i, S, u} \times \Delta_{j, S, u}^{*}:(B(t)-\sqrt{u} c t)+\beta\left(B^{*}(s)-\sqrt{u} c s\right)>\sqrt{u}\right\}, \\
p\left(i, j ; i^{\prime}, j^{\prime}\right)=\mathbb{P}\left\{\exists t, s \in \Delta_{i, S, u} \times \Delta_{j, S, u}^{*}:(B(t)-\sqrt{u} c t)+\beta\left(B^{*}(s)-\sqrt{u} c s\right)>\sqrt{u},\right. \\
\left.\exists t, s \in \Delta_{i^{\prime}, S, u} \times \Delta_{j^{\prime}, S, u}^{*}:(B(t)-\sqrt{u} c t)+\beta\left(B^{*}(s)-\sqrt{u} c s\right)>\sqrt{u}\right\}
\end{array}
$$

for $-N_{u} \leq i \leq N_{u}, 0 \leq j \leq N_{u}$, fixed $S>0$ and

$$
\Delta_{j, S, u}^{*}=\left[\frac{j S}{u}, \frac{j(S+1)}{u}\right] .
$$

By Bonferroni inequality

$\sum_{0 \leq j \leq N_{u}-1,-N_{u} \leq i \leq N_{u}-1} p(i, j)-\sum_{0 \leq j, j^{\prime} \leq N_{u},-N_{u}-1 \leq i, i^{\prime} \leq N_{u},\left(i, i^{\prime}\right) \neq\left(j, j^{\prime}\right)} p\left(i, j ; i^{\prime}, j^{\prime}\right) \leq \zeta(u) \leq \sum_{0 \leq j \leq N_{u},-N_{u}-1 \leq i \leq N_{u}} p(i, j)$.

The term

$$
\sum_{0 \leq j, j^{\prime} \leq N_{u},-N_{u}-1 \leq i, i^{\prime} \leq N_{u},\left(i, i^{\prime}\right) \neq\left(j, j^{\prime}\right)} p\left(i, j ; i^{\prime}, j^{\prime}\right)
$$

is negligible by the proof of Theorem 2.1, Eq. [14] in [9] and consequently

$$
\zeta(u) \sim \sum_{0 \leq j \leq N_{u},-N_{u} \leq i \leq N_{u}} p(i, j), \quad u \rightarrow \infty .
$$

Next we approximate $p(i, j)$ uniformly. Recall, that $v^{2}=u, c_{i, S, v}=t_{u}+\frac{i S}{v^{2}}, \varphi_{i, v}$ is the density function of $\sqrt{c_{i, S, v}} \mathcal{N}$ and set $G_{j}=[j S,(j+1) S] \cap \delta \mathbb{Z}$. Denote also $[a, b]_{\alpha}=[a, b] \cap \alpha \mathbb{Z}$ for any real numbers $a \leq b$ and $\alpha>0$. We have

$$
\begin{aligned}
p(i, j)= & \mathbb{P}\left\{\exists(t, s) \in \Delta_{i, S, u} \times \Delta_{j, S, u}^{*}: B(t)-B\left(c_{i, S, v}\right)-c \sqrt{u}\left(t-c_{i, S, v}\right)+B\left(c_{i, S, v}\right)-\sqrt{u} c c_{i, S, v}\right. \\
& \left.+\beta\left(B^{*}(s)-\sqrt{u} c s\right)>\sqrt{u}\right\} \\
= & \int_{\mathbb{R}} \mathbb{P}\left\{\exists(t, s) \in[0, S / u]_{\delta / u} \times \Delta_{j, S, u}^{*}: B(t)-\sqrt{u} c t-\sqrt{u} c c_{i, S, v}+\beta\left(B^{*}(s)-\sqrt{u} c s\right)>\sqrt{u}-x\right\} \varphi_{i, v}(x) d x \\
= & \int_{\mathbb{R}} \mathbb{P}\left\{\exists(t, s) \in[0, S]_{\delta} \times G_{j}: \frac{B(t)}{v}-v c\left(c_{i, S, v}+\frac{t}{v^{2}}\right)+\beta\left(\frac{B^{*}(s)}{v}-\frac{c s}{v}\right)>v-x\right\} \varphi_{i, v}(x) d x
\end{aligned}
$$




$$
\begin{aligned}
& =\frac{1}{v} \int_{\mathbb{R}} \mathbb{P}\left\{\exists(t, s) \in[0, S]_{\delta} \times G_{j}: \frac{B(t)}{v}-v c\left(c_{i, S, v}+\frac{t}{v^{2}}\right)+\beta\left(\frac{B^{*}(s)}{v}-\frac{c s}{v}\right)>v-\left(v-\frac{x}{v}\right)\right\} \varphi_{i, v}\left(v-\frac{x}{v}\right) d x \\
& =\frac{1}{v} \int_{\mathbb{R}} \mathbb{P}\left\{\exists(t, s) \in[0, S]_{\delta} \times G_{j}: B(t)-c t+\beta\left(B^{*}(s)-c s\right)>x+v^{2} c c_{i, S, v}\right\} \varphi_{i, v}\left(v-\frac{x}{v}\right) d x \\
& =\frac{1}{v} \int_{\mathbb{R}} \mathbb{P}\left\{\exists(t, s) \in[0, S]_{\delta} \times G_{j}: B(t)-c t+\beta\left(B^{*}(s)-c s\right)>x\right\} \varphi_{i, v}\left(v\left(1+c c_{i, S, v}\right)-\frac{x}{v}\right) d x \\
& =\frac{e^{-\frac{v^{2}\left(1+c c_{i, S, v}\right)^{2}}{2 c_{i, S, v}}}}{v \sqrt{2 \pi c_{i, S, v}}} \int_{\mathbb{R}} W_{j}(x) \omega(i, S, x) d x,
\end{aligned}
$$

where

$$
W_{j}(x)=\mathbb{P}\left\{\exists(t, s) \in[0, S]_{\delta} \times G_{j}: B(t)-c t+\beta\left(B^{*}(s)-c s\right)>x\right\}
$$

and $\omega(i, S, x)$ is defined in (17). By Borell-TIS inequality for all $|i|,|j| \leq N_{u}$ (proof is in the Appendix)

$$
\int_{\mathbb{R}} W_{j}(x) \omega(i, S, x) d x \sim \int_{\mathbb{R}} W_{j}(x) e^{2 c x} d x, \quad u \rightarrow \infty .
$$

Next we have with $G_{j}^{*}=\left[2 j c^{2} S, 2(j+1) c^{2} S\right] \cap 2 c^{2} \delta \mathbb{Z}$

$$
\begin{aligned}
\int_{\mathbb{R}} W_{j}(x) e^{2 c x} d x & =\frac{1}{2 c} \int_{\mathbb{R}} \mathbb{P}\left\{\sup _{(t, s) \in[0, S]_{\delta} \times G_{j}}\left(2 c B(t)-2 c^{2} t+\beta\left(2 c B^{*}(s)-2 c^{2} s\right)\right)>2 c x\right\} e^{2 c x} d(2 c x) \\
& =\frac{1}{2 c} \int_{\mathbb{R}} \mathbb{P}\left\{\sup _{(t, s) \in[0, S]_{\delta} \times G_{j}}\left(\sqrt{2} B\left(2 c^{2} t\right)-2 c^{2} t+\beta\left(\sqrt{2} B^{*}\left(2 c^{2} s\right)-2 c^{2} s\right)\right)>x\right\} e^{x} d x \\
& =\frac{1}{2 c} \mathbb{E}\left\{\sup _{(t, s) \in\left[0,2 c^{2} S\right]_{2 c^{2} \delta} \times G_{j}^{*}} \exp \left(\sqrt{2} B(t)-t+\beta\left(\sqrt{2} B^{*}(s)-s\right)\right)\right\} \\
& =\frac{1}{2 c} \mathbb{E}\left\{\sup _{t \in\left[0,2 c^{2} S\right]_{2 c^{2} \delta}} e^{\sqrt{2} B(t)-t}\right\} \mathbb{E}\left\{\sup _{s \in G_{j}^{*}} e^{\beta\left(\sqrt{2} B^{*}(s)-s\right)}\right\} .
\end{aligned}
$$

By (21) combined with the line above we write

$$
\begin{aligned}
\zeta(u) & \sim \frac{1}{2 c} \mathbb{E}\left\{\sup _{t \in\left[0,2 c^{2} S\right]_{2 c^{2} \delta}} e^{\sqrt{2} B(t)-t}\right\} \\
& \times \sum_{0 \leq j \leq N_{u}} \mathbb{E}\left\{\sup _{s \in G_{j}^{*}} e^{\beta\left(\sqrt{2} B^{*}(s)-s\right)}\right\} \sum_{-N_{u} \leq i \leq N_{u}} \frac{e^{-\frac{v^{2}\left(1+c c_{i, S, v}\right)^{2}}{2 c_{i, S, v}}}}{v \sqrt{2 \pi c_{i, S, v}}}, \quad u \rightarrow \infty .
\end{aligned}
$$

As was shown in the proof of Theorem 1.1 as $u \rightarrow \infty$ and then $S \rightarrow \infty$

$$
\frac{1}{2 c} \mathbb{E}\left\{\sup _{t \in\left[0,2 c^{2} S\right]_{2 c^{2} \delta}} e^{\sqrt{2} B(t)-t}\right\} \sum_{-N_{u} \leq i \leq N_{u}} \frac{e^{-\frac{v^{2}\left(1+c c_{i, S, v}\right)^{2}}{2 c_{i, S, v}}}}{v \sqrt{2 \pi c_{i, S, v}}} \sim \mathcal{H}_{2 c^{2} \delta} e^{-2 c u} .
$$

We have as $S \rightarrow \infty$ (proof of the first line below is in the Appendix)

$$
\sum_{0 \leq j \leq N_{u}} \mathbb{E}\left\{\sup _{s \in G_{j}^{*}} e^{\beta\left(\sqrt{2} B^{*}(s)-s\right)}\right\} \sim \mathbb{E}\left\{\sup _{s \in\left[0,2 c^{2} S\right]_{2 c^{2} \delta}} e^{\beta(\sqrt{2} B(s)-s)}\right\}
$$




$$
\begin{aligned}
& =\int_{\mathbb{R}} \mathbb{P}\left\{\sup _{s \in\left[0,2 c^{2} S\right]_{2 c^{2} \delta}}\left(\sqrt{2} B\left(s \beta^{2}\right)-\frac{s \beta^{2}}{\beta}>x\right)\right\} e^{x} d x \\
& =\int_{\mathbb{R}} \mathbb{P}\left\{\sup _{s \in\left[0,2 c^{2} \beta^{2} S\right]_{2 c^{2} \beta^{2} \delta}}\left(\sqrt{2} B(s)-s\left(1+\frac{1-\beta}{\beta}\right)\right)>x\right\} e^{x} d x \\
& \rightarrow \mathcal{P}_{2 c^{2} \beta^{2} \delta}^{\frac{1-\beta}{\beta}}=\mathcal{P}_{2 c^{2}(1-\gamma)^{2} \delta}^{\frac{\gamma}{1-\gamma}} \in(0, \infty) .
\end{aligned}
$$

Combining the statement above with (23) and (24) we conclude

$$
\zeta(u) \sim \mathcal{P}_{2 c^{2}(1-\gamma)^{2} \delta}^{\frac{\gamma}{1-\gamma}} \mathcal{H}_{2 c^{2} \delta} e^{-2 c u}, \quad u \rightarrow \infty
$$

and hence by (20) the claim follows.

Proof of Theorem 4.1. The proof is similar to that of Theorem 1.1 and we use similar notation as therein. We have by (8)

$$
\mathcal{P}_{\delta}(u, T) \sim \mathbb{P}\left\{\sup _{t \in\left[T_{u}^{-}, T_{u}^{+}\right] \cap \delta \mathbb{Z}} \inf _{s \in[t, t+T] \cap \delta \mathbb{Z}} Z(s)>u\right\}=: \widetilde{P}_{\delta}(u), \quad u \rightarrow \infty
$$

if we show that $\widetilde{P}_{\delta}(u) \geq C e^{-2 c u}$. By the self-similarity of Brownian motion

$$
\begin{aligned}
\widetilde{P}_{\delta}(u) & \left.=\mathbb{P}\left\{\exists t \in\left[T_{u}^{-}, T_{u}^{+}\right] \cap \delta \mathbb{Z}: \forall s \in[t, t+T] \cap \delta \mathbb{Z} Z(s)>u\right)\right\} \\
& =\mathbb{P}\left\{\exists t \in\left[\frac{T_{u}^{-}}{u}, \frac{T_{u}^{+}}{u}\right] \cap \delta \mathbb{Z}: \forall s \in\left[t, t+\frac{T}{u}\right] \cap \frac{\delta}{u} \mathbb{Z} B(s)>\sqrt{u}(1+c s)\right\} .
\end{aligned}
$$

We choose the same partition $\Delta_{j, S, u},-N_{u} \leq j \leq N_{u}$ of the interval $\Delta_{u}=\left[\frac{T_{u}^{-}}{u}, \frac{T_{u}^{+}}{u}\right]$ as in the proof of Theorem 1.1. The Bonferroni inequality yields

$$
\widetilde{p}_{1}(S, u) \geq \widetilde{P}_{\delta}(u) \geq \widetilde{p}_{1}(S, u)-\widetilde{p}_{2}(S, u)
$$

where

$$
\widetilde{p}_{1}(S, u)=\sum_{j=-N_{u}-1}^{N_{u}} \widetilde{p}_{j, S, u}, \quad \widetilde{p}_{1}(S, u)=\sum_{j=-N_{u}}^{N_{u}-1} \widetilde{p}_{j, S, u}, \quad \widetilde{p}_{2}(S, u)=\sum_{-N_{u}-1 \leq j<i \leq N_{u}} \widetilde{p}_{i, j ; S, u}
$$

with

$$
\widetilde{p}_{j, S, u}=\mathbb{P}\left\{\sup _{t \in \Delta_{j, S, u}} \inf _{s \in\left[t, t+\frac{T}{u}\right] \cap \frac{\delta}{u} \mathbb{Z}} Z(s)>u\right\}
$$

and

$$
\widetilde{p}_{i, j ; S, u}=\mathbb{P}\left\{\sup _{t \in \Delta_{i, S, u}} \inf _{s \in\left[t, t+\frac{T}{u}\right] \cap \frac{\delta}{u} \mathbb{Z}} Z(s)>u, \sup _{t \in \Delta_{j, S, u}} \inf _{s \in\left[t, t+\frac{T}{u}\right] \cap \frac{\delta}{u} \mathbb{Z}} Z(s)>u\right\} .
$$

Clearly, $\widetilde{p}_{i, j ; S, u} \leq p_{i, j ; S, u}$ and hence

$$
\widetilde{p}_{2}(S, u) \leq p_{2}(S, u) .
$$

Thus, if we show that $\widetilde{p}_{1}(S, u) \sim C_{1} e^{-2 c u}$ we conclude that $\widetilde{p}_{2}(S, u)$ is negligible. We approximate each summand in $\widetilde{p}_{1}(S, u)$ uniformly. As in the proof of Theorem 1.1 we obtain

$$
\widetilde{p}_{j, S, u}=\frac{e^{-v^{2}\left(1+c c_{j, S, v}\right)^{2} /\left(2 c_{j, S, v}\right)}}{v \sqrt{2 \pi c_{j, S, v}}} \int_{\mathbb{R}} w(T, x) \omega(j, S, x) d x
$$


where

$$
w(T, x)=\mathbb{P}\left\{\sup _{t \in[0, S] \cap \delta \mathbb{Z}} \inf _{s \in[t, t+T] \cap \delta \mathbb{Z}} Z(s)>x\right\}
$$

and $\omega(j, S, x)$ is defined in (17). By Borell-TIS inequality (similarly the proof of (18)) it follows that

$$
\int_{\mathbb{R}} w(T, x) \omega(j, S, x) d x \rightarrow \int_{\mathbb{R}} w(T, x) e^{2 c x} d x, \quad u \rightarrow \infty .
$$

Next we have

$$
\begin{aligned}
\int_{\mathbb{R}} w(T, x) e^{2 c x} d x=\frac{1}{2 c} \mathbb{E}\left\{\sup _{t \in[0, S] \cap \delta \mathbb{Z}} \inf _{s \in t, t+T] \cap \delta \mathbb{Z}} e^{2 c B(s)-2 c^{2} s}\right\} & =\frac{1}{2 c} \mathbb{E}\left\{\sup _{t \in[0, S] \cap \delta \mathbb{Z} s \in[t, t+T] \cap \delta \mathbb{Z}} e^{\sqrt{2} B\left(2 c^{2} s\right)-2 c^{2} s}\right\} \\
& =\frac{1}{2 c} \mathbb{E}\left\{\sup _{t \in\left[0,2 c^{2} S\right] \cap 2 c^{2} \delta \mathbb{Z} s \in\left[t, t+2 c^{2} T\right] \cap 2 c^{2} \delta \mathbb{Z}} e^{\sqrt{2} B(s)-s} \inf \right\} .
\end{aligned}
$$

It follows with similar arguments as in [24] that as $S \rightarrow \infty$

$$
\lim _{S \rightarrow \infty} \frac{1}{2 c^{2} S} \mathbb{E}\left\{\sup _{t \in\left[0,2 c^{2} S\right] \cap 2 c^{2} \delta \mathbb{Z}} \inf _{s \in\left[t, t+2 c^{2} T\right] \cap 2 c^{2} \delta \mathbb{Z}} e^{\sqrt{2} B(t)-t}\right\}=\mathcal{H}_{2 c^{2} \delta, 2 T c^{2}} \in(0, \infty),
$$

where the constant $\mathcal{H}_{2 c^{2} \delta, 2 T c^{2}}$ is given by (14). Hence by (19) we have

$$
\widetilde{P}_{\delta}(u) \sim \mathcal{H}_{2 c^{2} \delta, 2 T c^{2}} e^{-2 c u}, \quad u \rightarrow \infty
$$

and (26) holds, establishing the claim.

Proof of Theorem 4.2. We use below the same notation as in the previous proofs. By (8) we have

$$
\mathcal{C}_{\delta}(u, k) \sim \mathbb{P}\left\{\#\left\{t \in G(\delta) \cap\left(T_{u}^{-}, T_{u}^{+}\right): Z(t)>u\right\}>k\right\}=: \hat{\psi}_{k}^{\delta}(u), \quad u \rightarrow \infty
$$

if we show that $\hat{\psi}_{k}^{\delta}(u) \geq C e^{-2 c u}$. Using the self-similarity of Brownian motion for any $u>0$

$$
\hat{\psi}_{k}^{\delta}(u)=\mathbb{P}\left\{\#\left\{t \in\left(-\frac{\ln u}{\sqrt{u}}+t_{0}, t_{0}+\frac{\ln u}{\sqrt{u}}\right) \cap G\left(\frac{\delta}{u}\right): \frac{B(t)}{c t+1}>\sqrt{u}\right\}>k\right\} .
$$

Letting

$$
A_{j, u}:=\#\left\{t \in \Delta_{j, S, u}: Z(t)>u\right\}
$$

we have using the idea from [18]

$$
\begin{aligned}
\hat{\psi}_{\delta}^{k}(u) \leq & \mathbb{P}\left\{\sum_{j=-N_{u}-1}^{N_{u}} A_{j, u}>k\right\} \\
= & \mathbb{P}\left\{\sum_{j=-N_{u}-1}^{N_{u}} A_{j, u}>k,\left\{\text { there exists only one } j \text { such that } A_{j, u}>0\right\}\right\} \\
& +\mathbb{P}\left\{\sum_{j=-N_{u}-1}^{N_{u}} A_{j, u}>k,\left\{\text { there exists } i \neq j \text { such that } A_{i, u}>0 \text { and } A_{j, u}>0\right\}\right\} \\
=: & p_{1, k}(u)+\Pi_{0}(u) .
\end{aligned}
$$

On the other hand

$$
\hat{\psi}_{k}^{\delta}(u) \geq \mathbb{P}\left\{\sum_{j=-N_{u}}^{N_{u}-1} A_{j, u}>k\right\}
$$




$$
\begin{aligned}
& =\mathbb{P}\left\{\sum_{j=-N_{u}}^{N_{u}-1} A_{j, u}>k,\left\{\text { there exists only one } j \text { such that } A_{j, u}>0\right\}\right\} \\
& \quad+\mathbb{P}\left\{\sum_{j=-N_{u}}^{N_{u}-1} A_{j, u}>k,\left\{\text { there exists } i \neq j \text { such that } A_{i, u}>0 \text { and } A_{j, u}>0\right\}\right\} \\
& =: \quad p_{2, k}(u)+\Pi_{0}^{\prime}(u) .
\end{aligned}
$$

Notice, that $\Pi_{0}(u)$ and $\Pi_{0}^{\prime}(u)$ are less than the double-sum term in Theorem 1.1. They are negligible if we prove that $p_{2, k}(u) \sim p_{1, k}(u) \geq C e^{-2 c u}$ as $u \rightarrow \infty$ for some $C>0$. We have

$$
\begin{aligned}
p_{1, k}(u) & =\sum_{j=-N_{u}-1}^{N_{u}}\left(\mathbb{P}\left\{A_{j, u}>k\right\}-\mathbb{P}\left\{A_{j, u}>k, \exists i \neq j: A_{i, u}>0\right\}\right) \\
& =\sum_{j=-N_{u}-1}^{N_{u}} \mathbb{P}\left\{A_{j, u}>k\right\}-\sum_{j=-N_{u}-1}^{N_{u}} \mathbb{P}\left\{A_{j, u}>k, \exists i \neq j: A_{i, u}>0\right\} .
\end{aligned}
$$

The last summand is less than the double-sum term in Theorem 1.1 and is negligible. Thus we need to compute the asymptotic of

$$
Q_{\delta, k}(u):=\sum_{j=-N_{u}}^{N_{u}} \mathbb{P}\left\{A_{j, u}>k\right\} .
$$

With similar arguments as in the proof of Theorem 1.1

$$
\mathbb{P}\left\{A_{j, u}>k\right\}=\mathbb{P}\left\{\#\left\{t \in \Delta_{j, S, u}: Z(t)>u\right\}>k\right\}=\frac{e^{-v^{2}\left(1+c c_{j, S, v}\right)^{2} /\left(2 c_{j, S, v}\right)}}{v \sqrt{2 \pi c_{j, S, v}}} \int_{\mathbb{R}} w_{k}(x) \omega(j, S, x) d x,
$$

where $\omega(j, S, x)$ is defined in (17) and

$$
w_{k}(x)=\mathbb{P}\{\#\{t \in[0, S] \cap \delta \mathbb{Z}: Z(t)>x\}>k\} .
$$

Similarly to the proof of (18) we have

$$
\int_{\mathbb{R}} w_{k}(x) \omega(j, S, x) d x \rightarrow \int_{\mathbb{R}} w_{k}(x) e^{2 c x} d x, \quad u \rightarrow \infty .
$$

Next

$$
\int_{\mathbb{R}} w_{k}(x) e^{2 c x} d x=\frac{1}{2 c} \int_{\mathbb{R}} \mathbb{P}\left\{\#\left\{t \in\left[0,2 c^{2} S\right] \cap 2 c^{2} \delta \mathbb{Z}: \sqrt{2} B(t)-t>x\right\}>k\right\} e^{x} d x .
$$

As shown in [17]

$$
\lim _{S \rightarrow \infty} \frac{1}{2 c^{2} S} \int_{\mathbb{R}} \mathbb{P}\left\{\#\left\{t \in\left[0,2 c^{2} S\right] \cap 2 c^{2} \delta \mathbb{Z}: \sqrt{2} B(t)-t>x\right\}>k\right\} e^{x} d x=\mathcal{B}_{2 c^{2} \delta}(k) \in(0, \infty) .
$$

Consequently, by (19) as $u \rightarrow \infty$ and then $S \rightarrow \infty$

$$
Q_{\delta, k}(u) \sim c S \mathcal{B}_{2 c^{2} \delta}(k) \sum_{j=-N_{u}}^{N_{u}} \frac{e^{-v^{2}\left(1+c c_{j, S, v}\right)^{2} /\left(2 c_{j, S, v}\right)}}{v \sqrt{2 \pi c_{j, S, v}}}=\mathcal{B}_{2 c^{2} \delta}(k) e^{-2 c u} K_{v, S} \sim \mathcal{B}_{2 c^{2} \delta}(k) e^{-2 c u} .
$$

Since $\mathcal{B}_{2 c^{2} \delta}(k) \in(0, \infty)$, then $Q_{\delta, k}(u) \sim \hat{\psi}_{k}^{\delta}(u)$ as $u \rightarrow \infty$ implying

$$
\hat{\psi}_{k}^{\delta}(u) \sim e^{-2 c u} \mathcal{B}_{2 c^{2} \delta}(k), \quad u \rightarrow \infty .
$$




\section{Appendix}

Proof of (20). Recall,

$$
Z(t, s)=\frac{B(t)+(1-\gamma) B^{*}(s)}{c t+(1-\gamma) c s+1}, \quad s, t \geq 0
$$

where $B$ and $B^{*}$ are independent Brownian motions. For some positive $\varepsilon$ and large $u$ denote

$$
\begin{aligned}
A(\varepsilon) & =([0, \infty) \times[0, \infty)) \backslash\left(\left[-\varepsilon+t_{u}, \varepsilon+t_{u}\right] \times[0, \varepsilon]\right), \\
R(\varepsilon, u) & =\left(\left[-\varepsilon+t_{u}, \varepsilon+t_{u}\right] \times[0, \varepsilon]\right) \backslash\left(\left[-\frac{\ln u}{\sqrt{u}}+t_{u}, \frac{\ln u}{\sqrt{u}}+t_{u}\right] \times\left[0, \frac{\ln u}{\sqrt{u}}\right]\right) .
\end{aligned}
$$

We have

$$
\begin{aligned}
\mathbb{P}\left\{\exists(t, s) \in \mathbb{D}_{\delta}(u): Z(t, s)>\sqrt{u}\right\} & \leq \mathbb{P}\{\exists(t, s) \in G(\delta / u): Z(t, s)>\sqrt{u}\} \\
(32) & \leq \mathbb{P}\left\{\exists(t, s) \in \mathbb{D}_{\delta}(u): Z(t, s)>\sqrt{u}\right\}+\mathbb{P}\{\exists(t, s) \in A(\varepsilon): Z(t, s)>\sqrt{u}\} \\
& +\mathbb{P}\{\exists(t, s): R(\varepsilon, u): Z(t, s)>\sqrt{u}\} .
\end{aligned}
$$

We show that $Z(t, s)$ is a.s bounded for $t, s \geq 0$. According to Chapter 4, p. 31 in [25] it is equivalent that $Z(t, s)$ is bounded with positive probability. We have

$$
\begin{aligned}
\mathbb{P}\left\{\sup _{t, s \geq 0} Z(t, s) \leq 1\right\} & =\mathbb{P}\left\{\text { for all } t, s \geq 0 B(t)-c t+(1-\gamma)\left(B^{*}(s)-c s\right) \leq 1\right\} \\
& \geq \mathbb{P}\left\{\text { for all } t, s \geq 0 B(t)-c t \leq 1 / 2,(1-\gamma)\left(B^{*}(s)-c s\right) \leq 1 / 2\right\} \\
& =(1-\mathbb{P}\{\exists t \geq 0: B(t)-c t>1 / 2\})\left(1-\mathbb{P}\left\{\exists s \geq 0: B^{*}(s)-c s>1 / 2(1-\gamma)^{-1}\right\}\right) \\
& =\left(1-e^{-c}\right)\left(1-e^{-c(1-\gamma)^{-1}}\right)>0,
\end{aligned}
$$

where we used (1) for the last equation. Hence by Borell-TIS inequality (see [23])

$$
\mathbb{P}\{\exists(t, s) \in A(\varepsilon): Z(t, s)>\sqrt{u}\}=o(\mathbb{P}\{Z(1 / c, 0)>\sqrt{u}\}), \quad u \rightarrow \infty .
$$

Next we shall prove that

$$
\mathbb{P}\{\exists(t, s) \in R(\varepsilon, u): Z(t, s)>\sqrt{u}\}=o(\mathbb{P}\{Z(1 / c, 0)>\sqrt{u}\}), \quad u \rightarrow \infty .
$$

If we show that for any $(t, s) \in R(\varepsilon, u)$ and for some positive constant $C$ holds, that

$$
\sigma_{Z}^{2}(1 / c, 0)-\sigma_{Z}^{2}(t, s) \geq C \frac{\ln ^{2} u}{u}
$$

we can immediately claim (34) by Piterbarg's inequality (Proposition 9.2.5 in [25]). Notice that if i) $s \notin\left[0, \frac{\ln u}{\sqrt{u}}\right]$, then

$$
\begin{aligned}
\sigma_{Z}^{2}(1 / c, 0)-\sigma_{Z}^{2}(t, s) & =(A(1 / c, 0)-A(t, s))+A^{*}(t, s) \\
& \geq A^{*}(t, s)=\frac{s \beta(1-\beta)}{(c t+\beta c s+1)^{2}} \geq C \frac{\ln u}{\sqrt{u}} \geq C \frac{\ln ^{2} u}{u},
\end{aligned}
$$

hence the claim follows.

ii) assume that $s \in\left[0, \frac{\ln u}{\sqrt{u}}\right]$. Setting

$$
L(x)=\frac{x}{(c x+1)^{2}},
$$

we have that $L(x)$ attains its unique maxima at point $x=1 / c, L^{\prime}(1 / c)=0$ and $L^{\prime \prime}(1 / c)<0$. We have

$$
\sigma_{Z}^{2}(1 / c, 0)-\sigma_{Z}^{2}(t, s)=\sigma_{Z}^{2}(1 / c, 0)-A(t, s)+A^{*}(t, s) \geq \sigma_{Z}^{2}(1 / c, 0)-A(t, s)=L(1 / c)-L(t+\beta s) .
$$


For all $(t, s)$ such that $(t, s) \in R(\varepsilon, u), s \in\left[0, \frac{\ln u}{\sqrt{u}}\right]$ we have that $|1 / c-(t+\beta s)| \geq C \frac{\ln u}{\sqrt{u}}$. Hence

$$
L(1 / c)-L(t+\beta s) \geq C\left|L^{\prime \prime}(1 / c)\right|(1 / c-(t+\beta s))^{2} \geq C \frac{\ln ^{2} u}{u}
$$

and (34) holds.

Notice that for some positive constant $C$

$$
\mathbb{P}\left\{\exists t, s \in \mathbb{D}_{\delta}(u): Z(t, s)>\sqrt{u}\right\} \geq \mathbb{P}\left\{Z\left(t_{u}, 0\right)>\sqrt{u}\right\} \geq C \mathbb{P}\{Z(1 / c, 0)>\sqrt{u}\} .
$$

Combining the statement above with (32),(33) and (34) we establish (20).

Proof of (8). Notice that

$$
\mathbb{P}\left\{\sup _{t \notin\left[T_{u}^{-}, T_{u}^{+}\right]} Z(t)>u\right\} \leq \mathbb{P}\{\exists t, s \in A(\varepsilon): Z(t, s)>\sqrt{u}\}+\mathbb{P}\{\exists t, s \in R(\varepsilon, u): Z(t, s)>\sqrt{u}\},
$$

where $A(\varepsilon)$ and $R(\varepsilon, u)$ are defined in (31). Hence the claim follows by (33) and (34).

Proof of (21). We shall prove that

$$
\int_{\mathbb{R}} W_{j}(x) e^{-\frac{x^{2}}{2 v^{2} c_{i}}+x \frac{1+c c_{i}}{c_{i}}} d x=\int_{-M}^{M} W_{j}(x) e^{2 c x} d x+\bar{A}_{M, v}
$$

where $\bar{A}_{M, v} \rightarrow 0$ as $u \rightarrow \infty$ and then $M \rightarrow \infty$ uniformly for all $|i|,|j| \leq N_{u}$. We have

$$
\begin{aligned}
& \int_{\mathbb{R}} W_{j}(x) e^{-\frac{x^{2}}{2 v^{2} c_{i}}+x \frac{1+c c_{i}}{c_{i}}} d x-\int_{-M}^{M} W_{j}(x) e^{2 c x} d x \\
\leq & \int_{|x|>M} W_{j}(x) e^{-\frac{x^{2}}{2 u c_{i}}+x \frac{1+c c_{i}}{c_{i}}} d x+\left|\int_{-M}^{M} W_{j}(x) e^{2 c x}\left(e^{-\frac{x^{2}}{2 u c_{i}}-\frac{x}{u} \frac{\left(\theta_{u}+i S\right) c}{c_{i}}}-1\right) d x\right| \\
= & : \quad \bar{I}_{1}+\left|\bar{I}_{2}\right| .
\end{aligned}
$$

Let $u \geq M^{6}$. For any integer $|i|,|j| \leq N_{u}$, all $x \in[-M, M]$ and all $u$ large for some constant $C$ that does not depend on $u$ we have

$$
\left.\mid \frac{\left(\theta_{u}+i S\right) c x}{c_{i}}\right)|\leq C M \sqrt{u} \ln u, \quad| \frac{x^{2}}{2 c_{i}} \mid \leq C M^{2},
$$

hence

$$
\left|\frac{1}{u}\left(-\frac{x^{2}}{2 c_{i}}-\frac{\left(\theta_{u}+i S\right) c x}{c_{i}}\right)\right| \leq \frac{C}{u}\left(M^{2}+M \sqrt{u} \ln u\right) \leq \frac{C}{M^{4}}+\frac{C M}{u^{2 / 5}} \leq \frac{1}{M} .
$$

We have by (22) and (39) that for all $|j| \leq N_{u}$

$$
\int_{\mathbb{R}} W_{j}(x) e^{2 c x} d x \leq C
$$

for some constant $C$ that does not depend on $u$ and hence

$$
\left|\bar{I}_{2}\right| \leq \frac{1}{M} \int_{-M}^{M} W_{j}(x) e^{2 c x} d x \leq \frac{1}{M} \int_{\mathbb{R}} W_{j}(x) e^{2 c x} d x=\frac{C}{M} \rightarrow 0, \quad M \rightarrow \infty .
$$


Next we have for large $u$

$$
\begin{aligned}
\bar{I}_{1} & \leq \int_{|x|>M} \mathbb{P}\left\{\exists t \in[0, S], s \geq 0: B(t)-c t+\beta\left(B^{*}(s)-c s\right)>x\right\} e^{x \frac{1+c c_{i}}{c_{i}}} d x \\
& \leq \int_{x>M} \mathbb{P}\left\{\exists t \in\left[0, \frac{S}{x}\right], s \geq 0: Z(t, s)>\sqrt{x}\right\} e^{x \frac{1+c c_{i}}{c_{i}}} d x+\int_{x<-M} e^{c x} d x .
\end{aligned}
$$

We analyze behavior of $\sigma_{Z}^{2}(t, s)$ on the set $\left\{(t, s) \in\left[0, \frac{S}{x}\right] \times[0, \infty)\right\}$. Since

$$
\sigma_{Z}^{2}(t, s)=\frac{t+\beta^{2} s}{(c t+c \beta s+1)^{2}} \leq \frac{S}{x}+\frac{\beta^{2} s}{(c \beta s+1)^{2}} \leq \frac{S}{x}+\frac{\beta}{4 c}
$$

taking large enough $x$ we can write for any fixed $\varepsilon>0$ that

$$
\sigma_{Z}^{2}(t, s) \leq \frac{\beta(1+\varepsilon)}{4 c}
$$

Hence by Borell-TIS inequality for large $x$

$$
\mathbb{P}\left\{\exists t \in\left[0, \frac{S}{x}\right], s \geq 0: Z(t, s)>\sqrt{x}\right\} \leq e^{-x \frac{2 c}{\beta(1+2 \varepsilon)}} .
$$

Choosing $\varepsilon$ such that $\beta(1+2 \varepsilon)<1$, uniformly for all $|i|,|j| \leq N_{u}$ we have with $a=2 c-\frac{2 c}{\beta(1+2 \varepsilon)}<0$

$$
\begin{aligned}
\bar{I}_{1} \leq o(1)+\int_{|x|>M} e^{x \frac{1+c c_{i}}{c_{i}}-x \frac{2 c}{\beta(1+2 \varepsilon)}} d x & =o(1)+\int_{|x|>M} e^{x\left(2 c+o(1)-\frac{2 c}{\beta(1+2 \varepsilon)}\right)} d x \\
& \leq o(1)+2 \int_{|x|>M} e^{a x} \rightarrow 0, \quad M \rightarrow \infty .
\end{aligned}
$$

Combination of (37) and (38) establishes (35). By the monotone convergence theorem (35) implies (21).

Proof of (18). We have

$$
\int_{\mathbb{R}} w(x) \omega(j, S, x) d x-\int_{-M}^{M} w(x) e^{2 c x} d x \leq \int_{|x|>M} w(x) \omega(j, S, x) d x+\left|\int_{|x|<M} w(x)\left(\omega(j, S, x)-e^{2 c x}\right)\right| d x=: I_{1}+\left|I_{2}\right| .
$$

Since $W_{j}(x) \geq w(x)$ we have that $\left(\bar{I}_{1}\right.$ and $\bar{I}_{2}$ are defined in (36))

$$
\left|I_{2}\right| \leq\left|\bar{I}_{2}\right|, \quad I_{1} \leq \bar{I}_{1}
$$

implying

$$
I_{1}+I_{2} \leq \bar{I}_{1}+\left|\bar{I}_{2}\right| \rightarrow 0
$$

as $u \rightarrow \infty$ and then $M \rightarrow \infty$ by (37) and (38). Thus (18) is established.

Proof of $(25)$. For any $j \geq 1$ we have $\left(\right.$ set $\left.b_{j}=2 j c^{2} S\right)$

$$
\begin{aligned}
\mathbb{E}\left\{\sup _{s \in G_{j}^{*}} e^{\beta\left(\sqrt{2} B^{*}(s)-s\right)}\right\} & \leq \int_{\mathbb{R}} \mathbb{P}\left\{\sup _{s \in\left[2 j c^{2} S, 2(j+1) c^{2} S\right]} \beta\left(\sqrt{2} B(s)-s-\sqrt{2} B\left(b_{j}\right)+b_{j}\right)>x+\beta\left(b_{j}-\sqrt{2} B\left(b_{j}\right)\right)\right\} e^{x} d x \\
& =\frac{1}{\sqrt{2 \pi b_{j}}} \int_{\mathbb{R}} \int_{\mathbb{R}} \mathbb{P}\left\{\sup _{s \in\left[0,2 c^{2} S\right]} \beta(\sqrt{2} B(s)-s)>x+\beta\left(b_{j}-\sqrt{2} y\right)\right\} e^{x} d x e^{-\frac{y^{2}}{2 b_{j}}} d y
\end{aligned}
$$




$$
\begin{aligned}
& =\frac{1}{\sqrt{2 \pi b_{j}}} \int_{\mathbb{R}} \int_{\mathbb{R}} \mathbb{P}\left\{\sup _{s \in\left[0,2 c^{2} S\right]} \beta(\sqrt{2} B(s)-s)>x\right\} e^{x-\beta\left(b_{j}-\sqrt{2} y\right)} e^{-\frac{y^{2}}{2 b_{j}}} d x d y \\
& =\frac{e^{-\beta b_{j}}}{\sqrt{2 \pi b_{j}}} \int_{\mathbb{R}} \int_{\mathbb{R}} \mathbb{P}\left\{\sup _{s \in\left[0,2 c^{2} S\right]} \beta(\sqrt{2} B(s)-s)>x\right\} e^{x} e^{-\frac{y^{2}}{2 b_{j}}+\sqrt{2} \beta y} d x d y \\
& =\frac{e^{-\beta b_{j}}}{\sqrt{2 \pi b_{j}}} \int_{\mathbb{R}} \mathbb{P}\left\{\sup _{s \in\left[0,2 c^{2} S\right]} \beta(\sqrt{2} B(s)-s)>x\right\} e^{x} d x \int_{\mathbb{R}} e^{-\frac{y^{2}}{2 b_{j}}+\sqrt{2} \beta y} d y .
\end{aligned}
$$

For some constant $C$ that does not depend on $S$ we have by (1)

$$
\int_{\mathbb{R}} \mathbb{P}\left\{\sup _{s \in\left[0,2 c^{2} S\right]} \beta(\sqrt{2} B(s)-s)>x\right\} e^{x} d x \leq \int_{\mathbb{R}} \mathbb{P}\left\{\sup _{s \in[0, \infty)}\left(B(s)-\frac{s}{\beta \sqrt{2}}\right)>\frac{x}{\beta \sqrt{2}}\right\} e^{x} d x=1+\int_{0}^{\infty} e^{-x / \beta+x} \leq C .
$$

Since

$$
\int_{\mathbb{R}} e^{-\frac{y^{2}}{2 b_{j}}+\sqrt{2} \beta y} d y=\sqrt{2 \pi b_{j}} e^{\beta^{2} b_{j}}
$$

we have for some fixed small enough $\varepsilon$ and large $S$

$$
\mathbb{E}\left\{\sup _{s \in G_{j}^{*}} e^{\beta(\sqrt{2} B(s)-s)}\right\} \leq C e^{-\beta(1-\beta) b_{j}} \leq e^{-j S \varepsilon}
$$

Thus as $S \rightarrow \infty$

$$
\sum_{1 \leq j \leq N_{u}} \mathbb{E}\left\{\sup _{s \in G_{j}^{*}} e^{\beta(\sqrt{2} B(s)-s)}\right\} \leq e^{-\varepsilon S}(1+o(1))
$$

establishing the claim.

Acknowledgments: Author would like to thank the anonymous referee for the useful remarks. Partial financial supported by SNSF Grant 200021-175752/1 and PSG 1250 grant (Unil - St. Petersburg) is kindly acknowledged.

\section{REFERENCES}

[1] D. Iglehart, "Diffusion approximations in collective risk theory," Journal of Applied Probability, vol. 6, no. 4, pp. 285-292, 1969.

[2] K. Dębicki and M. Mandjes, Queues and Lévy fluctuation theory. Springer, 2015.

[3] V. I. Piterbarg and I. A. Kozik, "High excursions of Gaussian nonstationary processes in discrete time," 2018.

[4] A. B. Dieker and B. Yakir, "On asymptotic constants in the theory of extremes for Gaussian processes," Bernoulli, vol. 20, no. 3, pp. 1600-1619, 2014.

[5] K. Dębicki and E. Hashorva, "On extremal index of max-stable stationary processes," Probability and Mathematical Statistics, vol. 37, no. 2, pp. 299-317, 2017.

[6] J. Hüsler and V. I. Piterbarg, "A limit theorem for the time of ruin in a Gaussian ruin problem," Stochastic Process. Appl., vol. 118, no. 11, pp. 2014-2021, 2008.

[7] Z. Michna, "Remarks on Pickands' theorem," Probab. Math. Statist., vol. 37, no. 2, pp. 373-393, 2017. 
[8] K. Dębicki and E. Hashorva, "On extremal index of max-stable stationary processes," Probab. Math. Statist., vol. 37, no. 2, pp. 299-317, 2017.

[9] E. Hashorva, L. Ji, and V. I. Piterbarg, "On the supremum of $\gamma$-reflected processes with fractional Brownian motion as input," Stochastic Process. Appl., vol. 123, no. 11, pp. 4111-4127, 2013.

[10] E. Hashorva and L. Ji, "Approximation of passage times of $\gamma$-reflected processes with FBM input," J. Appl. Probab., vol. 51, no. 3, pp. 713-726, 2014.

[11] P. Liu, E. Hashorva, and L. Ji, "On the $\gamma$-reflected processes with fBm input," Lith. Math. J., vol. 55, no. 3, pp. 402-412, 2015.

[12] K. Dȩbicki, E. Hashorva, and P. Liu, "Extremes of $\gamma$-reflected Gaussian process with stationary increments," ESAIM Probab. Stat., vol. 21, pp. 495-535, 2017.

[13] R. Loeffen, I. Czarna, and Z. Palowski, "Parisian ruin probability for spectrally negative Lévy processes.," 2013.

[14] K. Dȩbicki, E. Hashorva, and L. Ji, "Parisian ruin of self-similar Gaussian risk processes," J. Appl. Probab., vol. 52, no. 3, pp. 688-702, 2015.

[15] K. Dȩbicki, E. Hashorva, and L. Ji, "Parisian ruin over a finite-time horizon," Sci. China Math., vol. 59, no. 3, pp. 557-572, 2016.

[16] K. Dębicki, P. Liu, and Z. Michna, "Sojourn time of Gaussian processes with trend," arXiv:1810.10145v1, 2018.

[17] K. Dȩbicki, Z. Michna, and X. Peng, "Approximation of sojourn times of Gaussian processes," 2018.

[18] K. Dȩbicki, E. Hashorva, L. Ji, and T. Rolski, "Sojourn time of correlated Brownian motions with drift in a cone,"

[19] V. I. Piterbarg, Asymptotic methods in the theory of Gaussian processes and fields, vol. 148 of Translations of Mathematical Monographs. Providence, RI: American Mathematical Society, 1996. Translated from the Russian by V.V. Piterbarg, revised by the author.

[20] K. Dȩbicki, E. Hashorva, L. Ji, and T. Rolski, "Extremal behaviour of hitting a cone by correlated Brownian motion with drift," Accepted for publication in Stoch. Proc. Appl., 2018.

[21] H. Albrecher and C. Hipp, "Lundberg's risk process with tax," Bl. DGVFM, vol. 28, no. 1, pp. 13-28, 2007.

[22] L. Bai, K. Dębicki, E. Hashorva, and L. Luo, "On generalised Piterbarg constants," Meth. Comp. Appl. Probab, 2017, doi: 10.1007/s11009-016-9537-0.

[23] M. Lifshits, Lectures on Gaussian processes. Springer Briefs in Mathematics, Springer, Heidelberg, 2012.

[24] K. Dębicki, S. Engelke, and E. Hashorva, "Generalized Pickands constants and stationary max-stable processes," Extremes, vol. 20, no. 3, pp. 493-517, 2017.

[25] V. I. Piterbarg, Twenty Lectures About Gaussian Processes. Atlantic Financial Press London New York, 2015.

Grigori Jasnovidov, Department of Actuarial Science, University of Lausanne,, Unil-Dorigny, 1015 LauSANNE, SWitZERLAND

E-mail address: Grigori.Jasnovidov@unil.ch 